\title{
A New Hybrid PRP-MMSIS Conjugate Gradient Method and Its Application in Portfolio Selection
}

\author{
Sindy DeVila $^{1 *}$, MaUlana MaliK $^{1}$, Wed GiYarti $^{2}$ \\ ${ }^{1}$ Department of Mathematics, Universitas Indonesia, Depok 16424, Indonesia \\ ${ }^{2}$ Mathematics Education Study Program, Universitas Islam Negeri Sunan Kalijaga, Yogyakarta 55281, Indonesia \\ *Corresponding author: sindy@sci.ui.ac.id
}

\begin{abstract}
In this paper, we propose a new hybrid coefficient of conjugate gradient method (CG) for solving unconstrained optimization model. The new coefficient is combination of the PRP (PolakRibiére-Polyak) [1, 2] and a part of MMSIS (Malik-Mustafa-Sabariah-Ibrahim-Sukono) [3] coefficients. Under exact line search, the search direction of new method satisfies the sufficient descent condition and based on certain assumption, we establish the global convergence properties. Using some test functions, numerical results show that the proposed method is efficient than MMSIS method. Besides, the new method can be used to solve portfolio selection problem .
\end{abstract}

Keywords: Conjugate gradient method, Exact line search, Sufficient descent condition, Global convergence, Portfolio selection

\section{Introduction}

In this paper, we present a new hybrid coefficient of conjugate gradient (CG) method for solving unconstrained optimization problem

$$
\min f(x), x \in \mathbb{R}^{n},
$$

where $f: \mathbb{R}^{n} \rightarrow \mathbb{R}$ is continuously differentiable function and its gradient is defined by $g(x)=\nabla f(x)$. CG methods are among the effective methods for solving large-scale problems.

The conjugate gradient method works by constructing sequence $\left\{x_{k}\right\}$ with iterative formula

$$
x_{k+1}=x_{k}+\alpha_{k} d_{k}, k=0,1,2, \ldots,
$$

where $\alpha_{k}$ is the step size which in this paper, we use the rule of exact line search

$$
f\left(x_{k}+\alpha_{k} d_{k}\right):=\min _{\alpha \geq 0} f\left(x_{k}+\alpha d_{k}\right)
$$

and $d_{k}$ is the search direction formulated by

$$
d_{k}:= \begin{cases}-g_{k}, & \text { if } k=0, \\ -g_{k}+\beta_{k} d_{k-1}, & \text { if } k>0,\end{cases}
$$


where $\beta_{k}$ is the gradient conjugation coefficient which the researchers are currently making modifications to as a computational improvement of the existing method [4]. Some of the well-known conjugate gradient coefficients are the Hestenes-Stiefel (HS) [5], Polak-Ribiére-Polyak (PRP) [1, 2], Liu-Storey (LS) [6], Fletcher-Reeves (FR) [7], conjugate descent (CD) [8], and Dai-Yuan (DY) [9]. These coefficients are defined by the following formulas:

$$
\begin{aligned}
& \beta_{k}^{H S}=\frac{g_{k}^{T} y_{k-1}}{d_{k-1}^{T} y_{k-1}}, \quad \beta_{k}^{P R P}=\frac{g_{k}^{T} y_{k-1}}{\left\|g_{k-1}\right\|^{2}}, \quad \beta_{k}^{L S}=\frac{g_{k}^{T} y_{k-1}}{-g_{k-1}^{T} d_{k-1}}, \\
& \beta_{k}^{F R}=\frac{\left\|g_{k}\right\|^{2}}{\left\|g_{k-1}\right\|^{2}}, \quad \beta_{k}^{C D}=\frac{\left\|g_{k}\right\|^{2}}{-d_{k-1}^{T} g_{k-1}}, \quad \beta_{k}^{D Y}=\frac{\left\|g_{k}\right\|^{2}}{d_{k-1}^{T} y_{k-1}},
\end{aligned}
$$

where $y_{k-1}=g_{k}-g_{k-1}, g_{k}=g\left(x_{k}\right)$ and $\|\cdot\|$ is the Euclidean norm. One of the variants of this CG method is the hybrid CG method, which is defined as the coefficient is a combination of the existing CG coefficients. The popular for hybrid conjugate gradient method are Touati-Ahmed and Storey (TS) method [10], Hu and Storey (HuS) method [11], Gilbert and Nocedal (GN) method [12], and Dai and Yuan (hDY and LS-CD) method [13]:

$$
\begin{aligned}
\beta_{k}^{T S} & =\left\{\begin{array}{ll}
\beta_{k}^{P R P}, & \text { if } 0 \leq \beta_{k}^{P R P} \leq \beta_{k}^{F R}, \\
\beta_{k}^{F R}, & \text { otherwise }
\end{array},\right. \\
\beta_{k}^{H u S} & =\max \left\{0, \min \left\{\beta_{k}^{P R P}, \beta_{k}^{F R}\right\}\right\}, \\
\beta_{k}^{G N} & =\max \left\{-\beta_{k}^{F R}, \min \left\{\beta_{k}^{P R P}, \beta_{k}^{F R}\right\}\right\}, \\
\beta_{k}^{h D Y} & =\max \left\{0, \min \left\{\beta_{k}^{H S}, \beta_{k}^{D Y}\right\}\right\}, \\
\beta_{k}^{L S-C D} & =\max \left\{0, \min \left\{\beta_{k}^{L S}, \beta_{k}^{C D}\right\}\right\} .
\end{aligned}
$$

When proposing new methods, the researchers also show the sufficient descent condition and global convergence properties. This properties are characteristics of good computational. A method is said to fulfill the sufficient descent condition, if there exists a constant $c>0$ such that for all $k$ :

$$
g_{k}^{T} d_{k} \leq-c\left\|g_{k}\right\|^{2}
$$

and satisfies the global convergence properties, if

$$
\lim _{k \rightarrow \infty} \inf \left\|g_{k}\right\|=0
$$

For the FR method, Zoutendijk has proved the global convergence properties under the exact line search [14] and Al-Baali also established the global convergence properties under inexact line search [15]. The hybrid TS and HuS methods satisfies the descent condition and global convergence property under the inexact line search, and computational results are superior than the FR and PRP methods. For a description of other methods, we can see it in [3] and [16].

Recently, Malik et.al [3] have proposed the new coefficient of CG method, which it is modification of NPRP coefficient [17]. The new coefficient is symbolized by $\beta_{k}^{M M S I S}$ and defined as follows:

$$
\beta_{k}^{M M S I S}= \begin{cases}\frac{\left\|g_{k}\right\|^{2}-\frac{\left\|g_{k}\right\|}{\left\|g_{k-1}\right\|}\left|g_{k}^{T} g_{k-1}\right|-\left|g_{k}^{T} g_{k-1}\right|}{\left\|d_{k-1}\right\|^{2}} & , \text { if }\left\|g_{k}\right\|^{2}>\left(\frac{\left\|g_{k}\right\|}{\left\|g_{k-1}\right\|}+1\right)\left|g_{k}^{T} g_{k-1}\right|, \\ 0 & \text {, otherwise. }\end{cases}
$$

For the MMSIS method, the sufficient descent condition is satisfied under exact and strong line searches. Likewise, the MMSIS method satisfies the global convergence properties under exact and 
strong Wolfe line searches with parameter $\sigma \in(0,1 / 8)$. Numerical experiments shows that the MMSIS method is efficient than FR, CD, and DY methods. For other references about the CG method can refer to $[16,18,19,20,21,22,23,24]$.

Motivated by the MMSIS and GN methods, we propose a new hybrid CG coefficient for solving problem (1). The new coefficient is a combination of a part the MMSIS and PRP coefficients. Furthermore, we will establish the sufficient descent condition and global convergence properties under exact line search. Numerical experiments is also presented to compare the efficiency computational and the application of new method is used for solving portfolio selection problem. In the next section, we will present the formula of new coefficient, algorithm, sufficient descent condition, and global convergence properties. In Section 3, numerical experiments is provided and in Section 4, we show an application in portfolio selection. Finally, the conclusion is presented in Section 5.

\section{Algorithm and Convergence Analysis}

In this section, we formulate a new hybrid coefficient and establish the sufficient descent condition, and global convergence properties under exact line search. The new coefficient is a combination of part the MMSIS and PRP coefficients which formulated as follows:

$$
\beta_{k}^{H D M G}=\max \left\{\beta_{k}^{P R P}, \beta_{k}^{M M S I S *}\right\},
$$

where $\beta_{k}^{M M S I S *}=\frac{\left\|g_{k}\right\|^{2}-\frac{\left\|g_{k}\right\|}{\left\|g_{k-1}\right\|}\left|g_{k}^{T} g_{k-1}\right|-\left|g_{k}^{T} g_{k-1}\right|}{\left\|d_{k-1}\right\|^{2}}$, and HDMG denotes Hybrid-Devila-MalikGiyarti. The following algorithm describe the HDMG method.

Algorithm 1: (HDMG Method)

Step 1: Given initial point $x_{0} \in \mathbb{R}^{n}, d_{0}=-g_{0}$, stopping criteria $\varepsilon$, and set $k:=0$.

Step 2: If $\left\|g_{k}\right\| \leq \varepsilon$, then stop. $x_{k}$ is optimal point. Otherwise, go to next step.

Step 3: Compute $\beta_{k}^{H D M G}=\max \left\{\beta_{k}^{P R P}, \beta_{k}^{M M S I S *}\right\}$.

Step 4: Compute the search direction $d_{k}=-g_{k}+\beta_{k}^{H D M G} d_{k-1}$.

Step 5: Compute the step size $\alpha_{k}$ by using exact line search (3).

Step 6: Update new point for $k:=k+1$ by formula (2) and go to Step 2.

The following lemma show that the search direction $d_{k}$ under exact line search satisfies the sufficient descent condition.

Lemma 2.1. Suppose that a CG method with search direction (4), $\alpha_{k}$ is computed by using exact line search (3), and $\beta_{k}$ is computed by using (7), then, for all $k \geq 0$ the condition (5) is satisfied.

Proof. According to (4), we have $d_{0}=-g_{0}$, furthermore $g_{0}^{T} d_{0}=-g_{0} g_{0}=-\left\|g_{0}\right\|^{2}$. Thus, for $k=0$ the condition (5) fulfill. Now, for $k \geq 1$, we will show the condition (5) is satisfied. By multiplying (4) with $g_{k}^{T}$, we obtain

$$
g_{k}^{T} d_{k}=-g_{k}^{T} g_{k}+\beta_{k}^{H D M G} g_{k}^{T} d_{k-1}=-\left\|g_{k}\right\|^{2}+\beta_{k}^{H D M G} g_{k}^{T} d_{k-1} .
$$

Since $\alpha_{k}$ is computed by exact line search, it implies $g_{k}^{T} d_{k-1}=0$. Thus, we have $g_{k}^{T} d_{k}=-\left\|g_{k}\right\|^{2}$. Hence, the condition (5) fulfill. The proof is completed.

To establish the global convergence properties, we need to simplify the $\beta_{k}^{H D M G}$. See the following lemma.

Lemma 2.2. The value of $\beta_{k}^{H D M G}$ must be one of $\beta_{k}^{H D M G} \leq \frac{\left\|g_{k}\right\|^{2}}{\left\|d_{k-1}\right\|^{2}}$ or $\beta_{k}^{H D M G} \leq \frac{\left\|g_{k}\right\|^{2}}{\left\|g_{k-1}\right\|^{2}}$ or $\beta_{k}^{H D M G}=0$. 
ProOF. From (7), we have three cases.

- Case 1: if $\beta_{k}^{P R P}<\beta_{k}^{M M S I S *}$, we obtain

$$
\beta_{k}^{H D M G}=\beta_{k}^{M M S I S *}=\frac{\left\|g_{k}\right\|^{2}-\frac{\left\|g_{k}\right\|}{\left\|g_{k-1}\right\|}\left|g_{k}^{T} g_{k-1}\right|-\left|g_{k}^{T} g_{k-1}\right|}{\left\|d_{k-1}\right\|^{2}} \leq \frac{\left\|g_{k}\right\|^{2}}{\left\|d_{k-1}\right\|^{2}} .
$$

- Case 2: if $\beta_{k}^{P R P}>\beta_{k}^{M M S I S *}$, we obtain

$$
\beta_{k}^{H D M G}=\beta_{k}^{P R P}=\frac{g_{k}^{T}\left(g_{k}-g_{k-1}\right)}{\left\|g_{k-1}\right\|^{2}}=\frac{\left\|g_{k}\right\|^{2}-g_{k}^{T} g_{k-1}}{\left\|g_{k-1}\right\|^{2}} \leq \frac{\left\|g_{k}\right\|^{2}}{\left\|g_{k-1}\right\|^{2}} .
$$

- Case 3: if $\beta_{k}^{P R P}=\beta_{k}^{M M S I S *}=0$, we obtain

$$
\beta_{k}^{H D M G}=0
$$

The proof is finished.

The following assumption is needed to establish the convergence properties of HDMG method.

Assumption 2.3. (A1) The level set $\mathbb{Y}=\left\{x \in \mathbb{R}^{n}: f(x) \leq f\left(x_{0}\right)\right\}$ at $x_{0}$ is bounded. (A2) In any neighborhood $\mathbb{H}_{0}$ of $\mathbb{H}$, the objective function $f$ is differentiable and continuous, and its gradient $g(x)$ is Lipschitz continuous in $\mathbb{H}_{0}$, so, there exist a constant $L>0$ such that $\|g(x)-g(y)\| \leq L \| x-$ $y \|$, for all $x, y \in \mathbb{H}_{0}$.

Based on the assumption, Zoutendijk [14] has proven the following lemma which is necessary to prove the global convergence.

Lemma 2.4. Suppose that Assumption 2.3 hold. Consider any conjugate gradient method of the form (2) and (4), where $\alpha_{k}$ satisfy the exact line search (3). Then the following conditions, so, called Zoutendijk conditions hold:

$$
\sum_{k=0}^{\infty} \frac{\left(g_{k}^{T} d_{k}\right)^{2}}{\left\|d_{k}\right\|^{2}}<\infty
$$

The following theorem is global convergence theorem for HDMG method.

Theorem 2.5. Suppose that the sequence $\left\{x_{k}\right\}$ is generated by Algorithm 1. Assume that Assumption 2.3 hold. Then we have

$$
\liminf _{k \rightarrow \infty}\left\|g_{k}\right\|=0
$$

ProOf. Assume the opposite, i.e, (8) is not true, hence there exists a constant $z>0$ such that

$$
\left\|g_{k}\right\| \geq z, \forall k \geq 0
$$

it means that

$$
\frac{1}{\left\|g_{k}\right\|^{2}} \leq \frac{1}{z^{2}}, \forall k \geq 0,\left\|g_{k}\right\| \neq 0
$$

From (4), we know that

$$
d_{k}+g_{k}=\beta_{k}^{H D M G} d_{k-1} .
$$

By squaring both sides of the equation, we have

$$
\left\|d_{k}\right\|^{2}=\left(\beta_{k}^{H D M G}\right)^{2}\left\|d_{k-1}\right\|^{2}-2 \beta_{k}^{H D M G} g_{k}^{T} d_{k}-\left\|g_{k}\right\|^{2} .
$$


Dividing both sides of (10) by $\left(g_{k}^{T} d_{k}\right)^{2}$, we obtain

$$
\begin{aligned}
\frac{\left\|d_{k}\right\|^{2}}{\left(g_{k}^{T} d_{k}\right)^{2}} & =\frac{\left(\beta_{k}^{H D M G}\right)^{2}\left\|d_{k-1}\right\|^{2}}{\left(g_{k}^{T} d_{k}\right)^{2}}-\frac{2}{g_{k}^{T} d_{k}}-\frac{\left\|g_{k}\right\|^{2}}{\left(g_{k}^{T} d_{k}\right)^{2}} \\
& =\frac{\left(\beta_{k}^{H D M G}\right)^{2}\left\|d_{k-1}\right\|^{2}}{\left(g_{k}^{T} d_{k}\right)^{2}}-\left(\frac{1}{\left\|g_{k}\right\|}-\frac{\left\|g_{k}\right\|}{g_{k}^{T} d_{k}}\right)^{2}+\frac{1}{\left\|g_{k}\right\|^{2}} \\
& \leq \frac{\left(\beta_{k}^{H D M G}\right)^{2}\left\|d_{k-1}\right\|^{2}}{\left(g_{k}^{T} d_{k}\right)^{2}}+\frac{1}{\left\|g_{k}\right\|^{2}}
\end{aligned}
$$

According to Lemma 2.2, we have three cases:

- Case 1. if $\beta_{k}^{H D M G} \leq \frac{\left\|g_{k}\right\|^{2}}{\left\|d_{k-1}\right\|^{2}}$, then from (11) and Lemma 2.1, we obtain

$$
\frac{\left\|d_{k}\right\|^{2}}{\left(g_{k}^{T} d_{k}\right)^{2}} \leq \frac{\left\|g_{k}\right\|^{4}}{\left\|d_{k-1}\right\|^{4}} \frac{\left\|d_{k-1}\right\|^{2}}{\left(g_{k}^{T} d_{k}\right)^{2}}+\frac{1}{\left\|g_{k}\right\|^{2}}=\frac{1}{\left\|d_{k-1}\right\|^{2}}+\frac{1}{\left\|g_{k}\right\|^{2}}
$$

We know that $\frac{1}{\left\|d_{k}\right\|^{2}} \leq \frac{1}{\left\|g_{k}\right\|^{2}}$ (see Lemma 4 in [16]), then we get

$$
\frac{\left\|d_{k}\right\|^{2}}{\left(g_{k}^{T} d_{k}\right)^{2}} \leq \frac{1}{\left\|g_{k-1}\right\|^{2}}+\frac{1}{\left\|g_{k}\right\|^{2}}
$$

From (9) and the inequality above, we have

$$
\frac{\left\|d_{k}\right\|^{2}}{\left(g_{k}^{T} d_{k}\right)^{2}} \leq \frac{1}{z^{2}}+\frac{1}{z^{2}}=\frac{2}{z^{2}} .
$$

Furthermore,

$$
\sum_{k=0}^{n} \frac{\left(g_{k}^{T} d_{k}\right)^{2}}{\left\|d_{k}\right\|^{2}} \geq \sum_{k=0}^{n} \frac{z^{2}}{2}=\frac{n+1}{2} z^{2}
$$

By Taking $n \rightarrow \infty$, we get

$$
\sum_{k=0}^{\infty} \frac{\left(g_{k}^{T} d_{k}\right)^{2}}{\left\|d_{k}\right\|^{2}} \geq \lim _{n \rightarrow \infty} \frac{n+1}{2} z^{2}=+\infty
$$

This contradicts the Zoutendijk condition in Lemma 2.4. Hence, the HDMG method is global convergence.

- Case 2. if $\beta_{k}^{H D M G} \leq \frac{\left\|g_{k}\right\|^{2}}{\left\|g_{k-1}\right\|^{2}}$, then from (11) and Lemma 2.1, we obtain

$$
\frac{\left\|d_{k}\right\|^{2}}{\left(g_{k}^{T} d_{k}\right)^{2}} \leq \frac{\left\|g_{k}\right\|^{4}}{\left\|g_{k-1}\right\|^{4}} \frac{\left\|d_{k-1}\right\|^{2}}{\left(g_{k}^{T} d_{k}\right)^{2}}+\frac{1}{\left\|g_{k}\right\|^{2}}=\frac{\left\|d_{k-1}\right\|^{2}}{\left\|g_{k-1}\right\|^{4}}+\frac{1}{\left\|g_{k}\right\|^{2}} .
$$

By utilizing (12) recursively, we get

$$
\frac{\left\|d_{k}\right\|^{2}}{\left(g_{k}^{T} d_{k}\right)^{2}} \leq \sum_{i=0}^{k} \frac{1}{\left\|g_{i}\right\|^{2}}
$$


Furthermore, from (9), we have

$$
\frac{\left(g_{k}^{T} d_{k}\right)^{2}}{\left\|d_{k}\right\|^{2}} \geq \frac{z^{2}}{k+1} .
$$

By taking summation of both sides, we obtain

$$
\sum_{k=0}^{n} \frac{\left(g_{k}^{T} d_{k}\right)^{2}}{\left\|d_{k}\right\|^{2}} \geq \sum_{k=0}^{n} \frac{z^{2}}{k+1}=z^{2} \sum_{k=0}^{n} \frac{1}{k+1} .
$$

This implies,

$$
\sum_{k=0}^{\infty} \frac{\left(g_{k}^{T} d_{k}\right)^{2}}{\left\|d_{k}\right\|^{2}} \geq+\infty
$$

This contradicts the Zoutendijk condition in Lemma 2.4. Hence, the HDMG method is global convergence.

- Case 3. if $\beta_{k}^{H D M G}=0$, then from (11) and (9), we obtain

$$
\frac{\left\|d_{k}\right\|^{2}}{\left(g_{k}^{T} d_{k}\right)^{2}} \leq \frac{1}{\left\|g_{k}\right\|^{2}} \leq \frac{1}{z^{2}} .
$$

Therefore,

$$
\frac{\left(g_{k}^{T} d_{k}\right)^{2}}{\left\|d_{k}\right\|^{2}} \geq z^{2}
$$

Thus,

$$
\sum_{k=0}^{\infty} \frac{\left(g_{k}^{T} d_{k}\right)^{2}}{\left\|d_{k}\right\|^{2}} \geq+\infty
$$

This contradicts the Zoutendijk condition in Lemma 2.4. Hence, the HDMG method is global convergence.

\section{Numerical Experiments}

In this section, we report the numerical experiments of HDMG method to compare with MMSIS method. The comparing done by using some test functions considered by Andrei [25], and Jamil and Yang [26]. Every test function, we use several initial points, and dimensions from 2 to 10,000. Most of the starting points used were considered by Andrei [25] and the rest were randomly. The numerical results are presented in Table 1 and obtained with the MATLAB code R2019a, and run using personal laptop; Intel Core i7 processor, 16 GB RAM, 64 bit Windows 10 Pro operating system. The stopping criterion $\left\|\mathbf{g}_{k}\right\| \leq \varepsilon$, where $\varepsilon=10^{-6}$.

According to the numerical results in Table 1, we can compare between methods by illustrating the performance profile curves, in this paper we will use the performance profile proposed by Dolan and Moré [27]. We plot the performance profile curve using the formula as follows:

$$
r_{p, s}=\frac{a_{p, s}}{\min \left\{a_{p, s}: p \in P \text { and } s \in S\right\}}, \rho_{s}(\tau)=\frac{1}{n_{p}} \operatorname{size}\left\{p \in P: \log _{2} r_{p, s} \leq \tau\right\},
$$

where $r_{p, s}$ is the performance profile ratio used to compare the $s$ solver performance method with the best performance for any $p$ problem solver. $\rho_{s}(\tau)$ is the probability that the best possible ratio is a consideration for solvers. Generally, the best method is represented on the top curve. 
Table 1: Numerical results for the MMSIS and HDMG methods.

\begin{tabular}{|c|c|c|c|c|c|c|}
\hline \multirow[t]{2}{*}{ Test Functions } & \multirow[t]{2}{*}{ Dimensions } & \multirow[t]{2}{*}{ Initial Points } & \multicolumn{2}{|c|}{ MMSIS } & \multicolumn{2}{|c|}{ HDMG } \\
\hline & & & NOI & CPU & NOI & CPU \\
\hline Ext White \& Holst & 1,000 & $(-1.2,1, \ldots,-1.2,1)$ & 16 & 0.4396 & 11 & 0.2952 \\
\hline Ext White \& Holst & 1,000 & $(10, . ., 10)$ & 30 & 0.804 & 37 & 1.0595 \\
\hline Ext White \& Holst & 10,000 & $(-1.2,1, . .,-1.2,1)$ & 17 & 4.363 & 12 & 3.0189 \\
\hline Ext White \& Holst & 10,000 & $(5, \ldots, 5)$ & 25 & 6.3978 & 18 & 4.561 \\
\hline Ext Rosenbrock & 1,000 & $(-1.2,1, \ldots,-1.2,1)$ & 16 & 0.0754 & 21 & 0.0827 \\
\hline Extended Rosenbrock & 1,000 & $(10, \ldots, 10)$ & 30 & 0.1338 & 21 & 0.0716 \\
\hline Ext Rosenbrock & 10,000 & $(-1.2,1, \ldots,-1.2,1)$ & 16 & 0.2979 & 21 & 0.3561 \\
\hline Ext Rosenbrock & 10,000 & $(5, \ldots, 5)$ & 26 & 0.4768 & 11 & 0.2092 \\
\hline Ext Freudenstein \& Roth & 4 & $(0.5,-2,0.5,-2)$ & 9 & 0.0502 & 8 & 0.0277 \\
\hline Ext Freudenstein \& Roth & 4 & $(-5,-5,-5,-5)$ & 7 & 0.0348 & 5 & 0.0164 \\
\hline Ext Beale & 1,000 & $(1,0.8, \ldots, 1,0.8)$ & 13 & 0.4102 & 10 & 0.2804 \\
\hline Ext Beale & 1,000 & $(0.5, \ldots, 0.5)$ & 12 & 0.3768 & 10 & 0.2736 \\
\hline Ext Beale & 10,000 & $(-1, \ldots,-1)$ & 14 & 3.8664 & 9 & 2.5379 \\
\hline Ext Beale & 10,000 & $(0.5, \ldots, 0.5)$ & 12 & 3.3274 & 10 & 2.844 \\
\hline Ext Wood & 4 & $(-3,-1,-3,-1)$ & 203 & 0.4727 & 158 & 0.3283 \\
\hline Ext Wood & 4 & $(5,5,5,5)$ & 272 & 0.6224 & 278 & 0.5681 \\
\hline Raydan 1 & 10 & $(1, \ldots, 1)$ & 21 & 0.0777 & 17 & 0.0435 \\
\hline Raydan 1 & 10 & $(10, \ldots, 10)$ & 75 & 0.2111 & 39 & 0.101 \\
\hline Raydan 1 & 100 & $(-1, \ldots,-1)$ & 118 & 0.4247 & 73 & 0.2121 \\
\hline Raydan 1 & 100 & $(-10, \ldots,-10)$ & 194 & 0.6187 & 170 & 0.4862 \\
\hline Ext Tridiagonal 1 & 500 & $(2, \ldots, 2)$ & 12 & 0.215 & 13 & 0.2004 \\
\hline Ext Tridiagonal 1 & 500 & $(10, \ldots, 10)$ & 139 & 2.0098 & 16 & 0.2573 \\
\hline Ext Tridiagonal 1 & 1,000 & $(1, \ldots, 1)$ & 12 & 0.3666 & 13 & 0.3797 \\
\hline Ext Tridiagonal 1 & 1,000 & $(-10, \ldots,-10)$ & 198 & 5.2889 & 15 & 0.4877 \\
\hline Diagonal 4 & 500 & $(1, \ldots, 1)$ & 5 & 0.04 & 3 & 0.0185 \\
\hline Diagonal 4 & 500 & $(-20, \ldots,-20)$ & 5 & 0.0293 & 4 & 0.0273 \\
\hline Diagonal 4 & 1,000 & $(1, \ldots, 1)$ & 5 & 0.0347 & 3 & 0.0183 \\
\hline Diagonal 4 & 1,000 & $(-30, \ldots,-30)$ & 5 & 0.0386 & 4 & 0.0303 \\
\hline Ext Himmelblau & 1,000 & $(1, \ldots, 1)$ & 9 & 0.0654 & 7 & 0.0453 \\
\hline Ext Himmelblau & 1,000 & $(20, \ldots, 20)$ & 6 & 0.0429 & 6 & 0.0452 \\
\hline Ext Himmelblau & 10,000 & $(-1, \ldots,-1)$ & 10 & 0.227 & 9 & 0.1886 \\
\hline Ext Himmelblau & 10,000 & $(50, \ldots, 50)$ & 7 & 0.173 & 6 & 0.1399 \\
\hline FLETCHCR & 10 & $(0, \ldots, 0)$ & 80 & 0.2188 & 56 & 0.1301 \\
\hline FLETCHCR & 10 & $(10, \ldots, 10)$ & 39 & 0.1233 & 30 & 0.083 \\
\hline Ext Powel & 100 & $(3,-1,0,1, \ldots)$ & 810 & 3.7825 & 3307 & 14.6059 \\
\hline Ext Powel & 100 & $(5, \ldots, 5)$ & 264 & 1.3266 & 3088 & 14.4368 \\
\hline NONSCOMP & 2 & $(3,3)$ & 8 & 0.0442 & 9 & 0.0238 \\
\hline NONSCOMP & 2 & $(10,10)$ & 15 & 0.0628 & 14 & 0.0405 \\
\hline Extended DENSCHNB & 10 & $(1, \ldots, 1)$ & 7 & 0.0368 & 5 & 0.0143 \\
\hline Extended DENSCHNB & 10 & $(10, \ldots, 10)$ & 10 & 0.0489 & 9 & 0.0264 \\
\hline Extended DENSCHNB & 100 & $(10, \ldots, 10)$ & 11 & 0.0461 & 9 & 0.0292 \\
\hline Extended DENSCHNB & 100 & $(-50, \ldots,-50)$ & 11 & 0.0564 & 8 & 0.027 \\
\hline Extended Penalty & 10 & $(1,2, \ldots, 10)$ & 22 & 0.0824 & 27 & 0.0687 \\
\hline Extended Penalty & 10 & $(-10, \ldots,-10)$ & 8 & 0.0377 & 7 & 0.0228 \\
\hline Extended Penalty & 100 & $(5, \ldots, 5)$ & 13 & 0.0613 & 7 & 0.0246 \\
\hline
\end{tabular}


Table 1 - Continued

\begin{tabular}{|c|c|c|c|c|c|c|}
\hline \multirow[t]{2}{*}{ Test Functions } & \multirow[t]{2}{*}{ Dimensions } & \multirow[t]{2}{*}{ Initial Points } & \multicolumn{2}{|c|}{ MMSIS } & \multicolumn{2}{|c|}{ HDMG } \\
\hline & & & NOI & $\mathrm{CPU}$ & NOI & CPU \\
\hline Extended Penalty & 100 & $(-10, \ldots,-10)$ & 10 & 0.0401 & 9 & 0.0442 \\
\hline Hager & 10 & $(1, \ldots, 1)$ & 13 & 0.0552 & 12 & 0.0353 \\
\hline Hager & 10 & $(-10, \ldots,-10)$ & 18 & 0.0746 & 18 & 0.051 \\
\hline Extended Maratos & 10 & $(1.1,0.1, \ldots, 1.1,0.1)$ & 53 & 0.1465 & 35 & 0.1071 \\
\hline Extended Maratos & 10 & $(-1, \ldots,-1)$ & 22 & 0.0764 & 12 & 0.0537 \\
\hline Six Hump Camel & 2 & $(-1,2)$ & 7 & 0.0247 & 6 & 0.0284 \\
\hline Six Hump Camel & 2 & $(-5,10)$ & 6 & 0.0207 & 6 & 0.0314 \\
\hline Three Hump Camel & 2 & $(-1,2)$ & 9 & 0.0293 & 9 & 0.0433 \\
\hline Three Hump Camel & 2 & $(2,-1)$ & 11 & 0.0325 & 12 & 0.059 \\
\hline Booth & 2 & $(5,5)$ & 4 & 0.0135 & 3 & 0.0145 \\
\hline Booth & 2 & $(10,10)$ & 4 & 0.0155 & 3 & 0.0164 \\
\hline Trecanni & 2 & $(-1,0.5)$ & 1 & 0.0064 & 1 & 0.0056 \\
\hline Trecanni & 2 & $(-5,10)$ & 5 & 0.0175 & 5 & 0.0264 \\
\hline Zettl & 2 & $(-1,2)$ & 11 & 0.0375 & 10 & 0.0457 \\
\hline Zettl & 2 & $(10,10)$ & 11 & 0.0303 & 8 & 0.0382 \\
\hline Shallow & 1,000 & $(0, \ldots, 0)$ & 8 & 0.042 & 7 & 0.0453 \\
\hline Shallow & 1,000 & $(10, \ldots, 10)$ & 11 & 0.0525 & 9 & 0.0613 \\
\hline Shallow & 10,000 & $(-1, \ldots,-1)$ & 9 & 0.1707 & 8 & 0.2012 \\
\hline Shallow & 10,000 & $(-10, \ldots,-10)$ & 9 & 0.194 & 9 & 0.1779 \\
\hline Generalized Quartic & 1,000 & $(1, \ldots, 1)$ & 5 & 0.0231 & 6 & 0.0342 \\
\hline Generalized Quartic & 1,000 & $(20, \ldots, 20)$ & 6 & 0.0365 & 10 & 0.0409 \\
\hline Quadratic QF2 & 50 & $(0.5, \ldots, 0.5)$ & 87 & 0.1943 & 71 & 0.1626 \\
\hline Quadratic QF2 & 50 & $(30, \ldots, 30)$ & 78 & 0.1845 & 64 & 0.1541 \\
\hline Leon & 2 & $(2,2)$ & 25 & 0.0608 & 11 & 0.0294 \\
\hline Leon & 2 & $(8,8)$ & 18 & 0.0446 & 33 & 0.0812 \\
\hline Generalized Tridiagonal 1 & 10 & $(2, \ldots, 2)$ & 24 & 0.0679 & 22 & 0.0868 \\
\hline Generalized Tridiagonal 1 & 10 & $(10, \ldots, 10)$ & 29 & 0.0829 & 27 & 0.108 \\
\hline Generlized Tridiagonal 2 & 4 & $(1, \ldots, 1)$ & 4 & 0.013 & 4 & 0.0182 \\
\hline Generalized Tridiagonal 2 & 4 & $(10, \ldots, 10)$ & 11 & 0.0363 & 10 & 0.0432 \\
\hline POWER & 10 & $(1, \ldots, 1)$ & 102 & 0.2045 & 21 & 0.0867 \\
\hline POWER & 10 & $(10, \ldots, 10)$ & 129 & 0.2674 & 25 & 0.0855 \\
\hline Quadratic QF1 & 50 & $(1, \ldots, 1)$ & 69 & 0.1599 & 38 & 0.094 \\
\hline Quadratic QF1 & 50 & $(10, \ldots, 10)$ & 85 & 0.1955 & 41 & 0.1126 \\
\hline Quadratic QF1 & 500 & $(1, \ldots, 1)$ & 240 & 1.3077 & 131 & 0.585 \\
\hline Quadratic QF1 & 500 & $(-5, \ldots,-5)$ & 424 & 2.4118 & 137 & 0.6383 \\
\hline Ext Quad Penalty QP2 & 100 & $(1, \ldots, 1)$ & 41 & 0.1438 & 26 & 0.0882 \\
\hline Ext Quad Penalty QP2 & 100 & $(10, \ldots, 10)$ & 36 & 0.1196 & 26 & 0.0958 \\
\hline Ext Quad Penalty QP2 & 500 & $(10, \ldots, 10)$ & 94 & 0.7527 & 33 & 0.2865 \\
\hline Ext Quad Penalty QP2 & 500 & $(50, \ldots, 50)$ & 96 & 0.8037 & 26 & 0.2048 \\
\hline Ext Quad Penalty QP1 & 4 & $(1,1,1,1)$ & 9 & 0.0251 & 6 & 0.0191 \\
\hline Ext Quad Penalty QP1 & 4 & $(10,10,10,10)$ & 9 & 0.0354 & 9 & 0.0259 \\
\hline Quartic & 4 & $(10,10,10,10)$ & 114 & 0.2794 & 365 & 0.9105 \\
\hline Quartic & 4 & $(15,15,15,15)$ & 118 & 0.3283 & 197 & 0.4841 \\
\hline Matyas & 2 & $(1,1)$ & 1 & 0.0039 & 1 & 0.0065 \\
\hline Matyas & 2 & $(20,20)$ & 1 & 0.006 & 1 & 0.0049 \\
\hline Colville & 4 & $(2,2,2,2)$ & 357 & 0.6761 & 204 & 0.4159 \\
\hline
\end{tabular}


Table 1 - Continued

\begin{tabular}{ccccccc}
\hline Test Functions & Dimensions & Initial Points & \multicolumn{2}{c}{ MMSIS } & \multicolumn{2}{c}{ HDMG } \\
\hline Colville & & & NOI & CPU & NOI & CPU \\
\hline Dixon and Price & 4 & $(10,10,10,10)$ & 58 & 0.1358 & 98 & 0.2037 \\
Dixon and Price & 3 & $(1,1,1)$ & 15 & 0.0403 & 13 & 0.042 \\
Sphere & 3 & $(10,10,10)$ & 18 & 0.0482 & 49 & 0.116 \\
Sphere & 5,000 & $(1, \ldots, 1)$ & 1 & 0.0169 & 1 & 0.0114 \\
Sum Squares & 5,000 & $(10, \ldots, 10)$ & 1 & 0.0164 & 1 & 0.013 \\
Sum Squares & 50 & $(0,1, \ldots, 0,1)$ & 49 & 0.1473 & 26 & 0.0694 \\
& 50 & $(10, \ldots, 10)$ & 80 & 0.2309 & 42 & 0.1037 \\
\hline
\end{tabular}

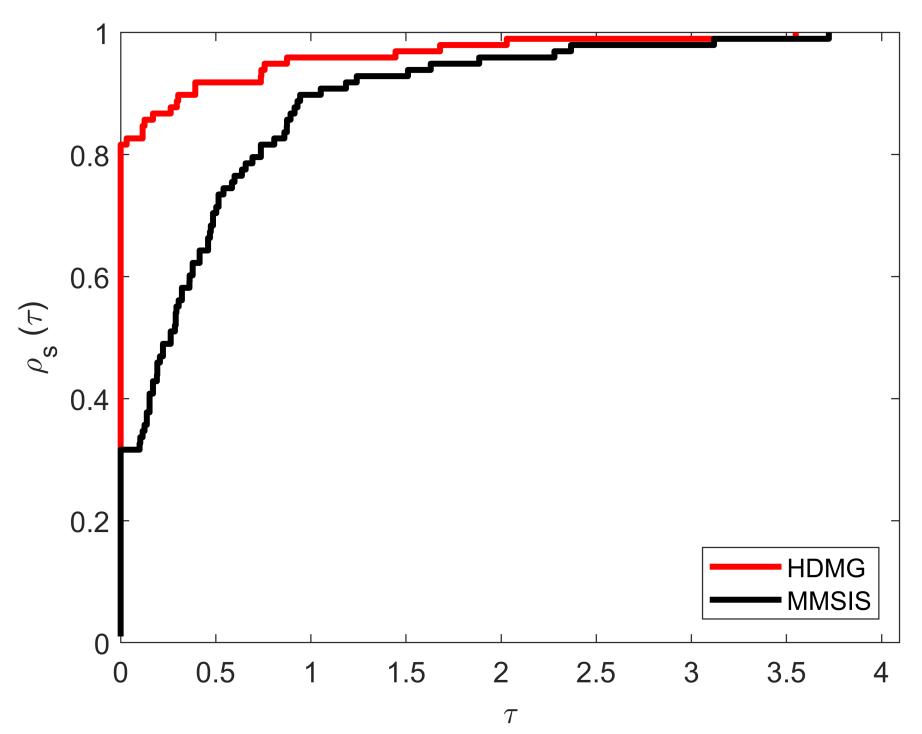

Figure 1: Performance Profile Based on Number of Iterations

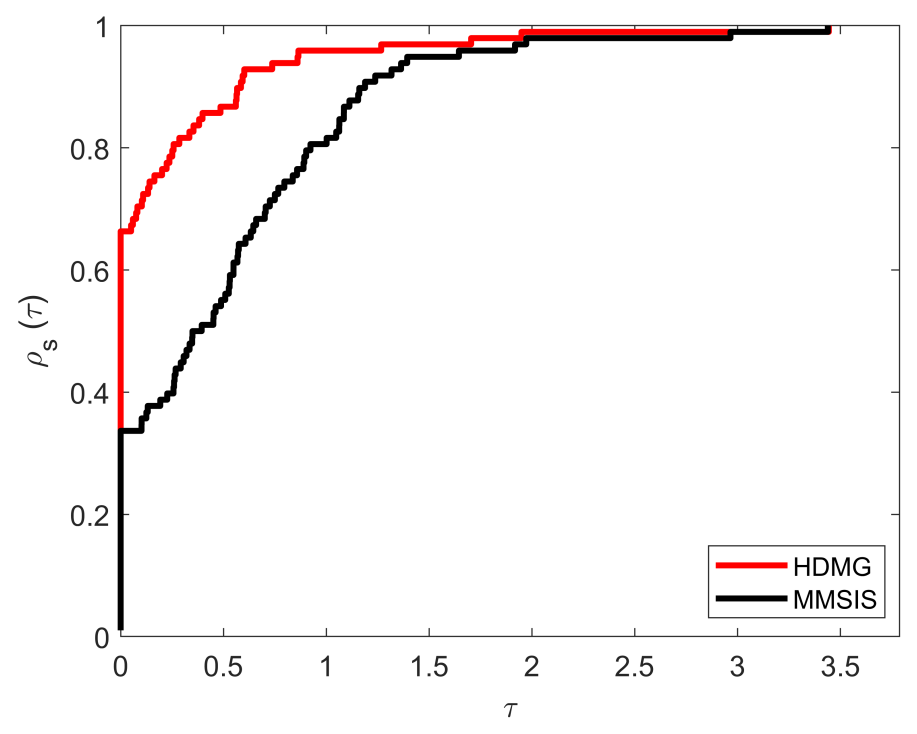

Figure 2: Performance Profile Based on CPU Time

In Fig. 1 and Fig. 2, the HDMG method is represented with red color, meanwhile, the MMSIS method is represented with black color. We can see that the curve of HDMG method always on the top MMSIS curve, so, based on Dolan and Moré rule, the HDMG method performs efficient than the MMSIS method both in terms of number of iterations and CPU time. 


\section{Application in Portfolio Selection}

In this section, we present the application of CG method for solving portfolio selection problem. Consider there are $M$ assets with return $r_{1}, \ldots, r_{M}$. Assume that expected return of asset denotes as $\mu^{T}=\left(\mu_{1}, \ldots, \mu_{M}\right)$ with $\mu_{i}=E\left[r_{i}\right], i=1, \ldots, M$, and covariance matrix denotes as $V=\left(\sigma_{i j}\right)$ with $\sigma_{i j}=\operatorname{Cov}\left(r_{i}, r_{j}\right), i, j=1, \ldots, M$. If proportional of asset is symbolized by $X^{T}=\left(x_{1}, x_{2}, \ldots, x_{m}\right)$, with subject to $\sum_{i=1}^{M}=1$, then, the expected return of portfolio is defined as follows:

$$
\mu_{p}=E\left[r_{p}\right]=\mu^{T} X
$$

and variance of portfolio is formulated by

$$
\sigma_{p}^{2}=\operatorname{Var}\left(r_{p}\right)=X^{T} V X
$$

In portfolio theory, many investors want to maximum returns or minimal risk or even both. Moreover, there are also extreme investors who only care about maximizing return (ignoring risk) or minimizing risk (ignoring expected returns) [28]. In this article we only consider minimizing the risks and using only two stocks from the database http://finance.yahoo.com, over a period of 3 years (Jan 1, 2018 Dec 31, 2020), i.e PT Bank Rakyat Indonesia (Persero) Tbk (BBRI), and PT Telekomunikasi Indonesia Tbk (TLKM). We just take the weekly closing price data and the return of each stock is defined as follows:

$$
R_{t}=\frac{P_{t}-P_{t-1}}{P_{t-1}},
$$

where $P_{t}$ is the stock prices at time $t$ and $P_{t-1}$ is the stock prices at time $t-1$. According to the data of return, we can plot the movement price as in Figure 3.

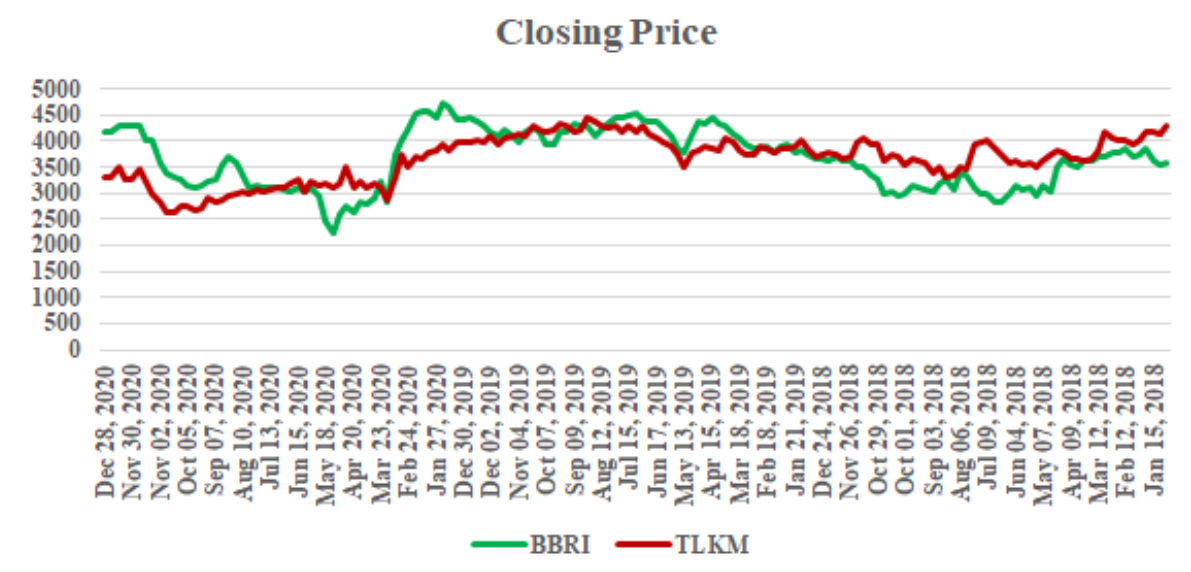

Figure 3: Closing Price of BBRI and TLKM in Currency IDR

The risk of our portfolio is defined as variance of the portfolio's return [28], so that the our problem can be written as:

$$
\left\{\begin{array}{l}
\text { minimize }: \sigma_{p}^{2}=X^{T} V X, \\
\text { subject to }: \sum_{j=1}^{2} x_{j}=1 .
\end{array}\right.
$$

We need to change the problem (13) into an unconstrained optimization problem. Suppose that $x_{2}=$ $1-x_{1}$, then the problem (13) is an unconstrained problem as follows:

$$
\min _{x_{1} \in \mathbb{R}}\left(\begin{array}{ll}
x_{1} & 1-x_{1}
\end{array}\right)^{T} V\left(\begin{array}{ll}
x_{1} & 1-x_{1}
\end{array}\right) .
$$

The value of mean, variance, and covariance for BBRI and TLKM stocks are presented in Table 2. 
Table 2: Mean, Variance and Covariance

\begin{tabular}{|c|c|c|c|c|c|}
\hline Stocks & Mean & Variance & Covariance & BBRI & TLKM \\
\hline BBRI & 0.00033 & 0.00273 & BBRI & 0.00273 & 0.00091 \\
\hline TLKM & 0.00247 & 0.00166 & TLKM & 0.00091 & 0.00166 \\
\hline
\end{tabular}

Based on Table 2, we can be compute the objective function of (14) as follows:

$$
f\left(x_{1}\right)=\left(0.00182 x_{1}+0.00091\right) x_{1}+\left(-0.00075 x_{1}+0.00166\right)\left(1-x_{1}\right)
$$

Now, we solve this function by using HDMG CG method with any initial points, then, we obtain $x_{1}=0.2916$. Furthermore, the value of risk is $\sigma_{p}^{2}=0.00144$. Finally, We found that to minimize the risk we have to invest $x_{1}=29.16 \%$ of the BBRI stock, and $x_{2}=70.84 \%$ of the TLKM stock. The portfolio risk is 0.00144 and the expected portfolio return is 0.0018 .

\section{Conclusion}

In this article, we presented a new hybrid CG method which is combination of PRP and a part of MMSIS coefficients. The new method satisfies the sufficient descent condition and global convergence properties under exact line search. Based on the numerical experiments, the new hybrid method is more efficient and robust than MMSIS method. Finally, the practical applicability of the hybrid method is also explored in risk optimization in portfolio selection problem.

\section{References}

[1] E. Polak and G. Ribiere, "Note sur la convergence de méthodes de directions conjuguées," Revue française d'informatique et de recherche opérationnelle. Série rouge, vol. 3, no. 16, pp. 35-43, 1969.

[2] B. T. Polyak, "The conjugate gradient method in extremal problems," USSR Computational Mathematics and Mathematical Physics, vol. 9, no. 4, pp. 94-112, 1969.

[3] M. Malik, M. Mamat, S. S. Abas, I. M. Sulaiman, and Sukono, "A new coefficient of the conjugate gradient method with the sufficient descent condition and global convergence properties.," Engineering Letters, vol. 28, no. 3, pp. 704-714, 2020.

[4] J. Nocedal and S. Wright, Numerical optimization. Springer Science \& Business Media, 2006.

[5] M. R. Hestenes and E. Stiefel, "Methods of conjugate gradients for solving linear systems," Journal of research of the National Bureau of Standards, vol. 49, no. 6, pp. 409-436, 1952.

[6] Y. Liu and C. Storey, "Efficient generalized conjugate gradient algorithms, part 1: theory," Journal of optimization theory and applications, vol. 69, no. 1, pp. 129-137, 1991.

[7] R. Fletcher and C. M. Reeves, "Function minimization by conjugate gradients," The computer journal, vol. 7, no. 2, pp. 149-154, 1964.

[8] R. Fletcher, Practical methods of optimization. John Wiley \& Sons, 2013.

[9] Y. Dai and Y. X. Yuan, "A nonlinear conjugate gradient method with a strong global convergence property," SIAM Journal on optimization, vol. 10, no. 1, pp. 177-182, 1999. 
[10] D. Touati-Ahmed and C. Storey, "Efficient hybrid conjugate gradient techniques," Journal of optimization theory and applications, vol. 64, no. 2, pp. 379-397, 1990.

[11] Y. Hu and C. Storey, "Global convergence result for conjugate gradient methods," Journal of Optimization Theory and Applications, vol. 71, no. 2, pp. 399-405, 1991.

[12] J. C. Gilbert and J. Nocedal, "Global convergence properties of conjugate gradient methods for optimization,” SIAM Journal on optimization, vol. 2, no. 1, pp. 21-42, 1992.

[13] Y.-h. Dai and Y. Yuan, "An efficient hybrid conjugate gradient method for unconstrained optimization," Annals of Operations Research, vol. 103, no. 1, pp. 33-47, 2001.

[14] G. Zoutendijk, "Nonlinear programming, computational methods," Integer and nonlinear programming, pp. 37-86, 1970.

[15] M. Al-Baali, "Descent property and global convergence of the fletcher-reeves method with inexact line search," IMA Journal of Numerical Analysis, vol. 5, no. 1, pp. 121-124, 1985.

[16] M. Malik, M. Mamat, S. S. Abas, I. M. Sulaiman, and Sukono, "Performance analysis of new spectral and hybrid conjugate gradient methods for solving unconstrained optimization problems.," IAENG International Journal of Computer Science, vol. 48, no. 1, pp. 66-79, 2021.

[17] L. Zhang, "An improved wei-yao-liu nonlinear conjugate gradient method for optimization computation," Applied Mathematics and computation, vol. 215, no. 6, pp. 2269-2274, 2009.

[18] I. Sulaiman, M. Mamat, M. Waziri, U. Yakubu, and M. Malik, "The convergence properties of a new hybrid conjugate gradient parameter for unconstrained optimization models," in Journal of Physics: Conference Series, vol. 1734, p. 012012, IOP Publishing, 2021.

[19] M. Malik, M. Mamat, S. S. Abas, et al., "Convergence analysis of a new coefficient conjugate gradient method under exact line search," International Journal of Advanced Science and Technology, vol. 29, no. 5, pp. 187-198, 2020.

[20] M. Malik, M. Mamat, S. S. Abas, I. M. Sulaiman, et al., "A new modification of nprp conjugate gradient method for unconstrained optimization," Advances in Mathematics: Scientific Journal, vol. 9, no. 7, pp. 4955-4970, 2020.

[21] M. Malik, S. S. Abas, M. Mamat, I. S. Mohammed, et al., "A new hybrid conjugate gradient method with global convergence properties," International Journal of Advanced Science and Technology, vol. 29, no. 5, pp. 199-210, 2020.

[22] M. Malik, M. Mamat, S. S. Abas, I. M. Sulaiman, et al., "A new spectral conjugate gradient method with descent condition and global convergence property for unconstrained optimization," J. Math. Comput. Sci., vol. 10, no. 5, pp. 2053-2069, 2020.

[23] I. Sulaiman, M. Mamat, M. Waziri, U. Yakubu, and M. Malik, "The performance analysis of a new modification of conjugate gradient parameter for unconstrained optimization models," Mathematics and Statistics, vol. 9, no. 1, pp. 16-23, 2021.

[24] A. B. Abubakar, P. Kumam, M. Malik, P. Chaipunya, and A. H. Ibrahim, "A hybrid fr-dy conjugate gradient algorithm for unconstrained optimization with application in portfolio selection," AIMS Mathematics, vol. 6, no. 6, pp. 6506-6527, 2021.

[25] N. Andrei, Nonlinear Conjugate Gradient Methods for Unconstrained Optimization. Springer, 2020. 
[26] M. Jamil and X.-S. Yang, "A literature survey of benchmark functions for global optimisation problems," International Journal of Mathematical Modelling and Numerical Optimisation, vol. 4, no. 2, pp. 150-194, 2013.

[27] E. D. Dolan and J. J. Moré, "Benchmarking optimization software with performance profiles," Mathematical Programming, vol. 91, no. 2, pp. 201-213, 2002.

[28] S. Roman, Introduction to the mathematics of finance: from risk management to options pricing. Springer Science \& Business Media, 2004. 